\title{
Expanded CD1c+CD163+ DC3s population in synovial tissues is associated with disease progression of osteoarthritis
}

\section{Guowei Qiu}

Shanghai University of TCM: Shanghai University of Traditional Chinese Medicine

\section{Lu Meng}

Institut Pasteur of Shanghai Chinese Academy of Sciences

Jun Xie

Shanghai Guanghua Hospital of Integrated Traditional Chinese and Western Medicine

Hui Feng

Shanghai Guanghua Hospital of Integrated Traditional Chinese and Western Medicine

\section{Songtao Sun}

Shanghai Guanghua Hospital of Integrated Traditional Chinese and Western Medicine

\section{Chenxin Gao}

Shanghai Guanghua Hospital of Integrated Traditional Chinese and Western Medicine

\section{Sheng Zhong}

Shanghai Guanghua Hospital of Integrated Traditional Chinese and Western Medicine

\section{Xirui Xu}

Shanghai Guanghua Hospital of Integrated Traditional Chinese and Western Medicine

\section{Bingxin Kang}

The First Affiliated Hospital of Henan University of CM

\section{Hui Xu}

Henan University of Chinese Medicine

\section{Chi Zhao}

Shanghai University of TCM: Shanghai University of Traditional Chinese Medicine

\section{Lei Ran}

Shanghai University of TCM: Shanghai University of Traditional Chinese Medicine

\section{A Xinyu}

Shanghai University of TCM: Shanghai University of Traditional Chinese Medicine

\section{Bo Xu}

Shanghai University of TCM: Shanghai University of Traditional Chinese Medicine

\section{Xiaohui Meng}

Shanghai University of TCM: Shanghai University of Traditional Chinese Medicine 
Institut Pasteur of Shanghai Chinese Academy of Sciences

\section{Lianbo Xiao ( $\nabla$ xiao_lianbo@163.com )}

Guanghua Hospital: Shanghai Guanghua Hospital of Integrated Traditional Chinese and Western Medicine https://orcid.org/0000-0003-4226-6002

\section{Research Article}

Keywords: Osteoarthritis, Dendritic Cells, DC3, Synovium Fluid

Posted Date: January 24th, 2022

DOI: https://doi.org/10.21203/rs.3.rs-1250384/v1

License: (a) This work is licensed under a Creative Commons Attribution 4.0 International License. Read Full License 


\section{Expanded CD1c+CD163+ DC3s population in synovial tissues is} associated with disease progression of osteoarthritis (3)

Guowei Qiu ${ }^{1}$, Lu Meng ${ }^{3} \dagger$, Jun Xie ${ }^{2,4}$, Hui Feng ${ }^{2,4}$, Songtao Sun ${ }^{2,4}$, Chenxin Gao ${ }^{2,4}$, Sheng Zhong ${ }^{2,4}$, Xirui $\mathrm{Xu}^{2,4}$, Bingxin Kang ${ }^{5}$, Hui $\mathrm{Xu}^{6}$, Chi Zhao ${ }^{1}$, Lei Ran ${ }^{1}$, Xinyu A ${ }^{1}$, Bo Xu ${ }^{1}$, Xiaohui Meng ${ }^{1}$, Xiaoming Zhang ${ }^{3 *}$, Lianbo Xiao ${ }^{1,2,4 *}$

1 Shanghai University of Traditional Chinese Medicine, Shanghai, China 2 Department of Orthopedics, Guanghua Hospital affiliated to Shanghai University of Traditional Chinese Medicine, Shanghai, China

3 The Center for Microbes, Development and Health, Key Laboratory of Molecular Virology \& Immunology, Institut Pasteur of Shanghai, Chinese Academy of Sciences/University of Chinese Academy of Sciences, Shanghai, China 4 Arthritis Institute of Integrated Traditional Chinese and Western Medicine, Shanghai Academy of Traditional Chinese Medicine, Shanghai University of Traditional Chinese Medicine, Shanghai, 200052, China

5 Department of Rehabilitation, The First affiliated Hospital of Henan University of Chinese Medicine, Zhengzhou, China

6 Henan University of Traditional Chinese Medicine, Zhengzhou, China

*Correspondence: xiao_lianbo@163.com Tel.: +86-021- 62805833

xmzhang@ips.ac.cn Tel.: +86-021-54923130

†Guowei Qiu and Lu Meng contributed equally to this work. 
23 Conflict of Interest: The authors declare that they have no conflict of interest.

24 


\section{Abstract}

Objective: The mechanisms underlying osteoarthritis (OA) have recently been hypothesized to involve a dysfunctional immune system. This study aimed to evaluate the landscape of immune cells infiltrating synovial tissue and to determine their function in OA progression.

Design: Synovial tissue, synovium fluid (SF) and peripheral blood were collected from 21 patients with OA. Mononuclear cells were isolated and characterized using flow cytometry. H\&E staining and multiplex immunohistochemistry (mIHC) histological assessment of synovial samples were performed. Cytokine levels in the SF were measured using ELISA.

Results: We observed similar frequencies of immune cells in the synovium and SF, which were enriched in macrophages, T cells, and DCs. Notably, DC3s, CD1c DC163 ${ }^{+}$ DCs, an inflammation-induced subpopulation of DCs, was significantly expanded in the synovium and SF. Furthermore, we found that DC3s were primarily located within the ectopic lymphoid-like structure (ELLS) in close proximity to $\mathrm{CD} 8^{+} \mathrm{T}$ cells. Finally, the level of TNF- $\alpha$ and IL12p70 in the SF correlated with the severity of OA, suggesting a possible link between DC3s and OA progression.

Conclusion: These data suggest that $\mathrm{OA}$ is an immune system-related disease and that DC3s may play an active role in OA progression by promoting ELLS formation and inflammatory responses.

Keywords: Osteoarthritis, Dendritic Cells, DC3, Synovium Fluid

\section{Introduction}


Osteoarthritis (OA) is the most prevalent arthritic condition in the elderly (over 60 years worldwide), and thus has a high impact on patient activity, and is associated with heavy economic burden ${ }^{1}$. Pain is the most common sign of disease and the leading cause of disability $^{2}$. Although OA is mainly considered a degradative condition of the articular cartilage, there is increasing evidence demonstrating that $\mathrm{OA}$ is a low-grade inflammatory disease that affects all tissues of the joint, characterized by profound changes in intracellular mechanisms, and decreased efficiency of the immune system with ageing ${ }^{3}$.

After $\mathrm{T}$ cells, macrophages, and other immune cells infiltrate into joint tissues, cytokines and chemokines such as TNF- $\alpha$ and IFN- $\gamma$ are secreted, the complement system is activated, and cartilage-degrading factors such as matrix metalloproteinases are released, causing damage to the articular cartilage ${ }^{4}$. There has been considerable success in the treatment of rheumatoid arthritis using anti-cytokine therapies ${ }^{5}$. However, these therapies did not show much effect in OA, highlighting the more complex nature of OA pathogenesis. Thus, a better understanding of the pathogenic mechanisms of chronic immune activation and the development of novel therapeutic strategies for OA are urgently required.

Dendritic cells (DCs) are a class of bone marrow-derived cells arising from lymphomyeloid hematopoiesis. DCs form an essential interface between the innate sensing of pathogens and the activation of adaptive immunity ${ }^{6}$. The initiation and control of immune responses depends on three major subsets: plasmacytoid DCs (pDCs), myeloid/conventional DC1 (cDC1s), and DC2 (cDC2s). Previously, we 
demonstrated that both macrophages and DCs were enriched in the joint synovium, suggesting that the type, density, and location of immune cells within the local milieu may strongly influence OA pathogenesis. Here, we aimed to evaluate and explore the full spectrum of immune cell types and DC subsets using high-dimensional flow cytometry and multiplex immunohistochemistry (mIHC). Specifically, cDC2s can be further separated into two subpopulations according to BTLA and CD163 expression: $\mathrm{BTLA}^{+} \mathrm{CD} 163^{-} \mathrm{cDC} 2 \mathrm{~s}$ and BTLA ${ }^{-} \mathrm{CD} 163^{+} \mathrm{DC} 3 \mathrm{~s}$, with the latter primarily induced by inflammation ${ }^{7}$. In the current study, significantly increased levels of inflammatory DC3s were observed in the synovium compared with PBMCs and synovial fluid (SF). In addition, $\mathrm{DC} 3 \mathrm{~s}$ and $\mathrm{CD}^{+} \mathrm{T}$ cells co-localized within ectopic lymphoid-like structures (ELLS), suggesting a role for DC3s in organizing ELLS by attracting and activating $\mathrm{CD}^{+} \mathrm{T}$ cells. Moreover, the number of ELLS is proportional to disease severity. Together, this study revealed that DC3s were highly enriched in the joints of OA patients, with potentially pathogenic roles in disease progression.

\section{Method}

\section{Study Population}

From March 2021 to June 2021, patients with OA in the Orthopedic Surgery Department of Guanghua Hospital, Shanghai University of Traditional Chinese Medicine (21 patients) were enrolled. The diagnostic criteria for OA conformed to those of the American College of Rheumatology. We used the Kellgren and Lawrence (K\&L) score to assess disease severity in patients with OA through imaging. Blood testing of 
each patient revealed that there was no inflammatory reaction in the body before the operation. The clinical characteristics of the study population are shown in Figure 1A. This study was approved by the Ethics Committee of Guanghua Hospital and all study participants provided written informed consent.

\section{Sample Preparation for Flow Cytometry Analysis}

Before surgery, $5 \mathrm{~mL}$ of venous whole blood from was collected OA patients, placed in a sodium heparin anticoagulation tube and transported at room temperature. During total knee arthroplasty (TKA) surgery, synovial tissue was collected, and the joint fluid was placed in EDTA tube, and transported on ice at $4^{\circ} \mathrm{C}$. Peripheral blood mononuclear cells (PBMCs) were separated by density centrifugation on Lymphoprep (Axis-Shield, Norway). For the flow cytometry assay, $100 \mu$ fluorescently labeled antibody mixture was added to each sample (containing CD16-BV510, CD33-BV711, CD56-PE, CD19BV650, CD14-A700, CD15/CD66b-PerCP-Cy5.5, CD1c-BB515, CD45-APC-Cy7, CD3-PE-Cy5.5, BTLA-PE-Cy5, CD88-PC594, CD141-APC, FceRI $\alpha-$ Biotin/Streptavidin -BUV395, CD123-BV786, CD163-BV605, and HLADR-BV421). Meanwhile, mononuclear cells (MNs) were obtained from tissues as follows: after rinsing tissue with $1 \times$ PBS three times, weighing, and first cutting into $1 \mathrm{~cm} \times 1 \mathrm{~cm}$ pieces with ophthalmological scissors for the mIHC assay, the remaining tissue was cut into small pieces. SF were frozen in aliquots of $100 \mu 1$ at $-80^{\circ} \mathrm{C}$ for subsequent cytokine detection; digestive enzymes (type II collagenase $10 \mathrm{mg} / \mathrm{mL}+\mathrm{DNaseI} 100 \mathrm{U} / \mathrm{mL}$ ) was then separately added to synovial tissue and SF, digested for $1 \mathrm{~h}$ in a $37^{\circ} \mathrm{C}$ shaker, and filtered through a $70 \mu \mathrm{m}$ mesh. Five ml of MACS buffer containing $1 \%$ FBS was then 
added, and the sample was centrifuged at $500 \times \mathrm{g}$ for $10 \mathrm{~min}$. The supernatant was discarded, $100 \mu \mathrm{L}$ of MACS buffer was added to resuspend the cells, the cells were transferred into a sterile EP tube, Zombie Yellow-BV570 was added for staining (RT, $30 \mathrm{~min}$ ), and $100 \mu \mathrm{l}$ fluorescently labeled antibody mixture was added for cell surface staining (room temperature, $15 \mathrm{~min}$ ). The cells were washed with $1 \mathrm{~mL}$ of MACS buffer $(800 \times \mathrm{g}, 2 \mathrm{~min})$ and the supernatant was discarded. Cells were then fixed in fixation buffer (eBioscience, Intracellular Fixation Buffer) for 10 min at $4{ }^{\circ} \mathrm{C}$, washed with 1 mL MACS buffer and resuspended in $500 \mu \mathrm{L}$ MACS buffer. A BD Fortessa flow analyzer and FlowJo software (V10.7.2) was used for data analysis. Synovial tissue samples were retrieved for histological analysis after paraffin embedding. Following hematoxylin and eosin (H\&E) staining of $4 \mu \mathrm{m}$-thick paraffinembedded sections, multiplex immunohistochemistry (mIHC) was performed according to the manufacturer's instructions (Thermo Fisher Scientific Logo, Opal ${ }^{\circledR}$ Kit). This process was performed using the following antibodies and fluorescent dyes in the following order: CD1c/Opal570, CD8/Opal520, and CD163/Opal650. Detailed procedures for mIHC and quantitative analysis were performed as previously reported ${ }^{9}$.

131 Slides were scanned and imaged using the PerkinElmer Vectra3 ${ }^{\circledR}$ platform and 132 analyzed in batches using PerkinElmer inform and R script for the quantification of 133 positively stained cells.

4. Cytokine detection

The joint fluid was thawed at room temperature for $10 \mathrm{~min}$. Before use, the joint fluid 
was centrifuged at $10,000 \times \mathrm{g}$ for $10 \mathrm{~min}$ and the supernatant was collected. ELISA

137 assays for human TNF- $\alpha$, IL12p70, or IL23 were performed according to the 138 manufacturer's instructions (Absin Bioscience Inc., abs510012/abs510006/abs510013).

139 The corresponding cytokine concentrations in each sample were obtained by referring 140 to the standard curve.

141 5. Statistical analysis

142 Continuous variables were compared using the t-test or non-parametric Mann-

143 Whitney U test, as appropriate. The Kruskal-Wallis test was used for the analysis of 144 immune cell frequencies between the study groups. In the latter case, variations in 145 statistical significance were further subjected to post-hoc pairwise analyses. The Mann-

146 Whitney $U$ test was performed to assess differences in cytokine expression in SF 147 samples. All the reported p-values were two-tailed. A p-value $<0.05$ was considered to 148 show a statistically significant difference. Statistical analyses were performed using 149 GraphPad Prism 8.3.0 software and SPSS 25.0. The number of nearest neighbors was 150 calculated on the assigned coordinates of each cell using $\mathrm{R}$ software with the spatstat 151 package.

\section{Differences in immune-cell patterns between synovium tissue, SF and PBMCs} in OA patients.

OA patients undergoing total knee arthroplasty (TKA) were enrolled in our study. 
signs. Of the 21 patients identified, the mean age was 71 years, with no symptoms of inflammation (Figure 1A). A total of $17 / 21$ patients with OA were female, consistent with the fact that autoimmune disease patients are most commonly female ${ }^{10}$. For each patient, mononuclear cells from the tissues (MNs) and peripheral blood (PBMCs) were isolated, as shown in Figure 1B.

To characterize the immune status of the synovium, we used 18-marker highdimensional flow cytometry to study the major immune cell populations compared to paired PBMCs and SFs. Nine immune cell subsets were annotated: neutrophils (SSC$\mathrm{H}^{\text {hi }} \mathrm{CD} 15^{+} \mathrm{CD} 16^{+}$), eosinophils (SSC-H $\left.{ }^{\text {hi }} \mathrm{CD} 15^{+}\right)$, basophils (FceRIa $\left.{ }^{+} \mathrm{HLADR}^{-}\right)$, T cells $\left(\mathrm{CD}^{+}\right), \mathrm{B}$ cells $\left(\mathrm{CD} 19^{+}\right)$, NK cells $\left(\mathrm{CD} 3^{-} \mathrm{CD} 56^{+}\right)$, NKT cells $\left(\mathrm{CD}^{+} \mathrm{CD} 56^{+}\right)$, macrophages $\left(\mathrm{CD} 88^{+} \mathrm{CD} 14^{+}\right)$, and $\mathrm{DCs}\left(\mathrm{CD} 123^{+} \mathrm{HLADR}^{+} \mathrm{pDCs}, \mathrm{CD} 1 \mathrm{c}^{+} \mathrm{mDCs}\right.$, and $\left.\mathrm{CD} 141^{+} \mathrm{mDCs}\right)$. Fig. 1 shows the conventional manual gating strategy used to define these populations.

A comparative analysis was performed on the synovium, SF, and PBMCs. A similar pattern of immune cells was observed in the synovium and SF, which was completely different from that observed in PBMCs (Figure 1C-E). Although the number of each immune cell population varied greatly among patients, the frequency of macrophages showed major differences in synovium $\left(50.77 \% \pm 29.65 \%\right.$ of CD $45^{+}$cells; $\mathrm{P}=.0001)$ compared to PBMCs $(10.96 \% \pm 6.38)$ (Figure 1D, SFigure 2). The frequencies of total DCs in the SF and synovium were also significantly higher than those in the PBMCs, whereas fewer neutrophils and B cells were found. NK cells and NK T cells did not exhibit any major differences. However, a decreased percentage of 
T cells was observed in both the synovium and SF ( $\sim 25 \% \mathrm{CD} 45^{+}$cells $)$. Taken together,

181

182

183

184

185

186

187

188 these data showed that macrophages, T cells, and NK cells were enriched, whereas granulocytes were decreased in the synovium compared with PBMCs.

\section{High infiltration of $\mathrm{CD}^{+} \mathrm{c}^{+} \mathrm{CD} 163^{+} \mathrm{DC} 3 \mathrm{~s}$ in synovium.}

DCs comprise various subsets and exhibit distinct functions in autoimmune diseases ${ }^{11}$. The DC subgroups pDCs $\quad\left(\mathrm{CD} 123^{+}\right), \quad \mathrm{DC} 1 \mathrm{~s} \quad\left(\mathrm{CD} 123^{-} \mathrm{CD} 141^{+}\right), \quad \mathrm{DC}^{\mathrm{s}}$ $\left(\mathrm{CD}_{1} \mathrm{c}^{+} \mathrm{BTLA}^{+} \mathrm{CD} 163^{-}\right)$, and $\mathrm{DC} 3 \mathrm{~s}\left(\mathrm{CD} 1 \mathrm{c}^{+} \mathrm{BTLA}^{-} \mathrm{CD} 163^{+}\right)$were further investigated in patients with OA. Each DC subgroup was gated as shown in Figure 2A. In line with earlier reports, the percentage of DC1s or total DCs was significantly higher in the synovium and SF than in the $\mathrm{PBMC}^{12}$ group, whereas the percentage of pDCs did not differ between the three groups (Figure 2B). A similar proportion of DC2s was observed in SF and PBMCs. Intriguingly, DCs in the synovium were composed of almost $100 \%$ DC3s. Among CD45 ${ }^{+}$immune cells, $\sim 0.5 \%$ of DC $3 \mathrm{~s}$ were enriched in the synovium, while DC3 levels were found to be 10-fold higher in the SF. These results indicate that an increased number of cDCs accumulates during OA progression.

\section{DC3s organize into an ectopic lymphoid -like structure (ELLS) associated with}

\section{disease severity.}

To better explore the extent of synovitis and the contribution of cells to the degree of inflammation, we performed histopathological analysis of synovial tissue samples collected from KL3 and KL4 OA patients. As shown in the left H\&E image in Figure 
3A, the lining cells formed a single layer, and the synovial stroma showed normal cellularity with no inflammatory infiltrates. However, the presence of synovial lesions consistent with low-grade synovitis demonstrated an increase in the thickness of the lining layer and stromal cellularity, with the presence of a few, mostly perivascular lymphocytes. Comparatively, high-grade synovitis (Figure 3A, right picture) was distinguished by the presence of a greatly thickened lining, increased infiltration of numerous lymphocytes, and typical ELLS.

Next, we applied mIHC to further investigate cellular infiltration in the OA synovium by simultaneously gating for CD1c, CD8, and CD163. CD1c ${ }^{+} \mathrm{CD} 163^{+} \mathrm{DC} 3 \mathrm{~s}$ and $\mathrm{CD}^{+} \mathrm{T}$ cells were readily detected (Figure 3B). Recently, DC3s was proved in vivo to efficiently induce differentiation of $\mathrm{CD} 8^{+} \mathrm{T}$ cells, while $\mathrm{DC} 3$ infiltration was found to correlate with $\mathrm{CD}^{+} \mathrm{T}$ cells accumulation in breast tumors ${ }^{7}$. Consistent with this, we observed that DC3s and $\mathrm{CD}^{+} \mathrm{T}$ cells were highly enriched in ELLS. We hypothesized that ELLS might be organized by DC3s, together with $\mathrm{CD} 8^{+} \mathrm{T}$ cells. To confirm this, we applied cellular phenotyping of the fluorescence image and depicted the spatial location of DC3s and $\mathrm{CD} 8^{+} \mathrm{T}$ cells in situ (SFigure 3). We divided the DC3s-CD8 ${ }^{+} \mathrm{T}$ cell distance as $<20 \mu \mathrm{m}$ and $>20 \mu \mathrm{m}{ }^{9}$. The relative number of DC3s was calculated and significantly higher numbers of DC3s were recovered within $20 \mu \mathrm{m}$ than beyond (Figure 3C). In particular, ELLS were only found in $<20 \mu \mathrm{m}$ positive samples including OA18, OA38, and OA39. These data suggest that the aggregation of DC3s was positively related to the formation of the ELLS structure.

DC3s are an important source of pro-inflammatory cytokines, including TNF- $\alpha$, 

SF of KL4 patients were significantly higher than those in KL3. Together, these results suggest that the increased infiltration of DC3s in joint infiltration, together with the elevated proinflammatory cytokines in SF, may play a critical role in the progression of OA.

\section{Discussion}

OA was initially defined as a disease induced by mechanical stress in the form of cartilage destruction, with minimal, if any, involvement of immune responses. Thus, OA, in contrast to rheumatoid arthritis (RA), has long been regarded as a noninflammatory disease ${ }^{13-15}$. However, recent studies have demonstrated that, at least in certain patients, $\mathrm{OA}$ is an inflammatory disease, with patients frequently found to exhibit inflammatory infiltration of synovial membranes ${ }^{16,17}$. Recent studies have shown that the number of inflammatory cells in synovial tissue is higher than that in peripheral blood ${ }^{18,19}$. Both macrophages and $\mathrm{T}$ cells play important roles in OA pathogenesis $^{20-23}$. Consistent with previous studies, we also found higher percentages of macrophages in synovial tissue and SF than in PBMCs, whereas a decreased number of $\mathrm{T}$ cells was measured in synovial tissue and $\mathrm{SF}^{16,24}$.

Surprisingly, among CD45 ${ }^{+}$cells, DCs were redundant in synovial tissue and SF.

DCs have the broadest range of antigen presentation. The activation of innate immunity plays a critical role in the development and progression of OA. Innate immunity, including inflammasome activation, is triggered by small endogenous molecules called damage-associated molecular patterns, which are released in the extracellular media 
after cell stress or damage, bind to pathogen-recognition receptors, including distinct Toll-like receptors, and activate the secretion of pro-inflammatory cytokines, resulting in joint inflammation. Moreover, the functional role of Toll-like receptors expressed on DCs in OA development has also been reported ${ }^{25}$. DC3s, defined as CD1 $\mathrm{c}^{+} \mathrm{CD} 163^{+}$cells, were significantly enriched in the synovium and could prime naïve $\mathrm{CD} 8^{+} \mathrm{T}$ cells. In synovial tissue, $\mathrm{CD} 8^{+} \mathrm{T}$ cells are often located at the periphery of the aggregation ${ }^{24}$. As shown by our mIHC results, a substantial number of DC3s and CD8 ${ }^{+} \mathrm{T}$ cells were observed within the synovium. Consistent with this, higher levels of TNF- $\alpha$ and IL12p70, which are mainly secreted by DC3, were measured in ELLS. Similar lymphoid-like structures, such as tertiary lymphoid organs in tumors, have been previously noted ${ }^{26,27}$. These structures are composed of various immune cell types, including dendritic cells and antigen-specific B-and T-lymphocytes. In contrast to secondary lymphoid organs, TLSs are not imprinted during embryogenesis, but are enrolled patients with OA experienced pain with severe joint deformation. This may be related to the increased infiltration of DC3s into the synovium, which would prime more $\mathrm{CD} 8^{+} \mathrm{T}$ cells, causing more serious damage to the joint. Indeed, increased levels of TNF- $\alpha$ and IL12p70, of which DC3s are a major source, were observed in the SF of 
play a critical role in OA pathogenesis, and that targeting DC3s might be a novel therapeutic target to treat OA in both the early and late phases.

\section{STATEMENTS \& DECLARATIONS}

\section{Acknowledgments}

This study was supported by Shanghai 13th Five-Year Key Specialized College Department of Osteoarthritis and Arthritis of Integrated Traditional Chinese and Western Medicine (shslczdzk04801).

\section{Contributions}

All authors contributed to the study conception and design. Material preparation, data collection and analysis were performed by [Lu Meng], [Guowei Qiu] and [Chenxin Gao]. The first draft of the manuscript was written by [Guowei Qiu] and [Lu Meng]. All authors commented on previous versions of the manuscript. All authors read and approved the final manuscript.

\section{Role of the funding source}

This study was supported by Shanghai 13th Five-Year Key Specialized College Department of Osteoarthritis and Arthritis of Integrated Traditional Chinese and Western Medicine (shslczdzk04801).

The authors declare that no funds, grants, or other support were received during the preparation of this manuscript.

\section{Competing interests}

The authors declare that they have no conflict of interest.

\section{Data Availability}


The datasets generated during and/or analysed during the current study are

291

292 available from the corresponding author on reasonable request.

\section{Ethics approval}

This is an observational study. The Ethics Committee of Guanghua Hospital has confirmed that no ethical approval is required.

\section{References}

[1] Hunter DJ, Bierma-Zeinstra S. Osteoarthritis. Lancet 393, 1745-1759, doi:10.1016/S01406736(19)30417-9 (2019).

[2] Katz JN, Arant KR, Loeser RF. Diagnosis and Treatment of Hip and Knee Osteoarthritis: A Review. JAMA 325, 568-578, doi:10.1001/jama.2020.22171 (2021).

[3] Robinson WH, Lepus CM, Wang Q, et al. Low-grade inflammation as a key mediator of the pathogenesis of osteoarthritis. Nat Rev Rheumatol 12, 580-592, doi:10.1038/nrrheum.2016.136 (2016).

[4] Woodell-May JE, Sommerfeld SD. Role of Inflammation and the Immune System in the Progression of Osteoarthritis. J Orthop Res 38, 253-257, doi:10.1002/jor.24457 (2020).

[5] Haseeb A, Haqqi TM. Immunopathogenesis of osteoarthritis. Clin Immunol 146, 185-196, doi:10.1016/j.clim.2012.12.011 (2013).

[6] Collin M, Bigley V. Human dendritic cell subsets: an update. Immunology 154, 3-20, doi:10.1111/imm.12888 (2018).

[7] Bourdely P, Anselmi G, Vaivode K. et al. Transcriptional and Functional Analysis of CD1c(+) Human Dendritic Cells Identifies a CD163(+) Subset Priming CD8(+)CD103(+) T Cells. Immunity 53, 335-352 e338, doi:10.1016/j.immuni.2020.06.002 (2020). 
[8] Villar J, Segura E. Decoding the Heterogeneity of Human Dendritic Cell Subsets. Trends Immunol 41, 1062-1071, doi:10.1016/j.it.2020.10.002 (2020).

[9] Ma J, Zheng B, Goswami S, et al. PD1(Hi) CD8(+) T cells correlate with exhausted signature and poor clinical outcome in hepatocellular carcinoma. J Immunother Cancer 7, 331, doi:10.1186/s40425-019-0814-7 (2019).

[10] Invernizzi P, Pasini S, Selmi C, Gershwin ME, Podda M. Female predominance and X chromosome defects in autoimmune diseases. J Autoimmun 33, 12-16, doi:10.1016/j.jaut.2009.03.005 (2009).

[11] Dutertre CA, Becht E, Irac SE, et al. Single-Cell Analysis of Human Mononuclear Phagocytes Reveals Subset-Defining Markers and Identifies Circulating Inflammatory Dendritic Cells. Immunity 51, 573-589 e578, doi:10.1016/j.immuni.2019.08.008 (2019).

[12] Ponchel F, Burska AN, Hensor EM, et al. Changes in peripheral blood immune cell composition in osteoarthritis. Osteoarthritis Cartilage 23, 1870-1878, doi:10.1016/j.joca.2015.06.018 (2015).

[13] Hügle T, Geurts J. What drives osteoarthritis?-synovial versus subchondral bone pathology. Rheumatology (Oxford) 56, 1461-1471, doi:10.1093/rheumatology/kew389 (2017).

[14] Li Y, Xiao W, Luo W, et al. Alterations of amino acid metabolism in osteoarthritis: its implications for nutrition and health. Amino Acids 48, 907-914, doi:10.1007/s00726-015-2168$\mathrm{x}(2016)$.

[15] Li Y, Luo W, Deng Z, Lei G. Diet-Intestinal Microbiota Axis in Osteoarthritis: A Possible Role. Mediators Inflamm 2016, 3495173, doi:10.1155/2016/3495173 (2016).

[16] Kuryliszyn-Moskal A. Comparison of blood and synovial fluid lymphocyte subsets in 
rheumatoid arthritis and osteoarthritis. Clin Rheumatol 14, 43-50, doi:10.1007/BF02208083 (1995).

[17] de Lange-Brokaar BJ, Ioan-Facsinay A, van Osch GJ, et al. Synovial inflammation, immune cells and their cytokines in osteoarthritis: a review. Osteoarthritis Cartilage 20, 14841499, doi:10.1016/j.joca.2012.08.027 (2012).

[18] Rollín R, Marco F, Jover JA, et al. Early lymphocyte activation in the synovial microenvironment in patients with osteoarthritis: comparison with rheumatoid arthritis patients and healthy controls. Rheumatol Int 28, 757-764, doi:10.1007/s00296-008-0518-7 (2008).

[19] Leheita O, Abed Elrazek NY, Younes S, Mahmoud AZ. Lymphocytes subsets in osteoarthritis versus rheumatoid arthritis. Egypt J Immunol 12, 113-124 (2005).

[20] Zhang H, Cai D, Bai X. Macrophages regulate the progression of osteoarthritis. Osteoarthritis Cartilage 28, 555-561, doi:10.1016/j.joca.2020.01.007 (2020).

[21] Chen Y, Jiang W, Yong H, et al. Macrophages in osteoarthritis: pathophysiology and therapeutics. Am J Transl Res 12, 261-268 (2020).

[22] Sakkas LI, Platsoucas CD. Role of T cells in the pathogenesis of osteoarthritis. Arthritis Rheum 46, 3112-3113, doi:10.1002/art.10483 (2002).

[23] Penatti A, Facciotti F, De Matteis R, et al. Differences in serum and synovial CD4+ T cells and cytokine profiles to stratify patients with inflammatory osteoarthritis and rheumatoid arthritis. Arthritis Res Ther 19, 103, doi:10.1186/s13075-017-1305-1 (2017).

[24] Haynes MK, Hume EL, Smith JB. Phenotypic characterization of inflammatory cells from osteoarthritic synovium and synovial fluids. Clin Immunol 105, 315-325, doi:10.1006/clim.2002.5283 (2002). 
[25] Nie F, Ding F, Chen B, Huang S, Liu Q, Xu C. Dendritic cells aggregate inflammation in experimental osteoarthritis through a toll-like receptor (TLR)-dependent machinery response to challenges. Life Sci 238, 116920, doi:10.1016/j.lfs.2019.116920 (2019).

[26] Hiraoka N, Ino Y, Yamazaki-Itoh R, Kanai Y, Kosuge T, Shimada K. Intratumoral tertiary lymphoid organ is a favourable prognosticator in patients with pancreatic cancer. Br J Cancer 112, 1782-1790, doi:10.1038/bjc.2015.145 (2015).

[27] N J, J T, S1 N, Gt B. Tertiary lymphoid structures and B lymphocytes in cancer prognosis and response to immunotherapies. Oncoimmunology 10, 1900508, doi:10.1080/2162402X.2021.1900508 (2021).

\section{Figure Legends}

Figure 1. Landscape of immune cells in synovium, SF and paired PBMC.

(A) 21 OA patients undergoing TKA were studied. (B) PBMC cells and tissue MNs were isolated separately from 21 OA donors. (C) Identification of 9 major immune cell subsets by tSNE of flow cytometry data of synovium, SF and PBMC using the FlowSOM algorithm. (D) Proportions of monocytes/macrophages, DCs, T cells and NK cells to $\mathrm{CD}^{4} 5^{+}$cells in PBMC, synovium and SF. Significance was assessed by Wilcoxon matched-pairs signed rank test. ***, $\mathrm{P}<0.001$. ****, $\mathrm{P}<0.0001$.

Figure 2. DC3s significantly infiltrated in synovium. (A) Gating strategy for identification of DC2s and DC3s in Flow Cytometry. (B) 4 DCs subsets were defined in PBMC, synovium and SF. (C) Histograms showing the proportion of DCs of CD45 cells in synovium comparing with PBMC or SF. Significance was assessed by Wilcoxon 
matched-pairs signed rank test. **, $\mathrm{P}<0.01$. ***, $\mathrm{P}<0.001 . * * * *, \mathrm{P}<0.0001$.

Figure 3. DC3s infiltrated in synovium is correlated with $\mathrm{CD} 8^{+} \mathrm{T}$ cells. (A) H\&E stain.

(B) Representative mIHC image shows the staining for CD1c (yellow), CD163 (red), CD8 (green) in the OA synovium. In left picture, white arrow indicates DC3s $\left(\mathrm{CD} 1 \mathrm{c}^{+} \mathrm{CD} 163^{+}\right)$. A typical ELLS consist plenty of DC3s and $\mathrm{CD} 8^{+} \mathrm{T}$ cells was showed in right image. Magnification $\times 200$. (C) Association of DC3s infiltration with $\mathrm{CD} 8^{+} \mathrm{T}$ cells. Increased number of DC3 were measure when $\mathrm{CD} 8^{+} \mathrm{T}$ cells located close to DC3s ( $<20$ um). Each dot represents a single data point; blue dots represent ELLS negative samples including OA37 and OA40, and violet dots represent ELLS positive ones. Histograms showing higher level of (D) IL12p70 and (E) TNF- $\alpha$ in severer OA patients. (F) No significant differences of IL23 were observed. Significance was assessed by Wilcoxon matched-pairs signed rank test. *, $\mathrm{P}<0.05$. **, $\mathrm{P}<0.01$.

Figure S1. Gating strategy used to define immune cell types by flow cytometry.

Figure S2. Proportions of each immune cell subsets to $\mathrm{CD}^{4} 5^{+}$cells in $\mathrm{PBMC}$, synovium and SF. Red line present $10 \% \mathrm{CD}^{+} 5^{+}$.

Figure S3. Distance analysis of $\mathrm{DC} 3 \mathrm{~s}$ and $\mathrm{CD}^{+} \mathrm{T}$ cell. Cellular phenotype of the fluorescence image depicted the spatial location of CD1c+CD163+ (red dots), CD163+(green dots) and CD8+(blue dots) in the synovium. Solid plots connected the nearest cells within $20 \mu \mathrm{m}$ from the CD1c+CD163+ and CD163+ to CD8+ respectively. 
Figure 1.

\begin{tabular}{lc} 
\\
\cline { 2 - 2 } Characteristic & data \\
\hline Number of patients(n) & 21 \\
Sex M/F & $4 / 17$ \\
Age years(Mean \pm SD) & $71.57 \pm 5.11$ \\
BMI kg/m $\left(\mathrm{m}^{2}(\right.$ Mean \pm SD) & $26.25 \pm 3.69$ \\
WBC $\times 10^{\circ} / \mathrm{L}($ Mean \pm SD) & $6.57 \pm 1.90$ \\
CRP mg/(Mean \pm SD) & $1.82 \pm 2.43$ \\
ESR mm/h(Mean \pm SD) & $11.62 \pm 7.86$ \\
K\&L Scores n(\%) & \\
III & $10(48)$ \\
IV & $11(52)$ \\
\hline
\end{tabular}
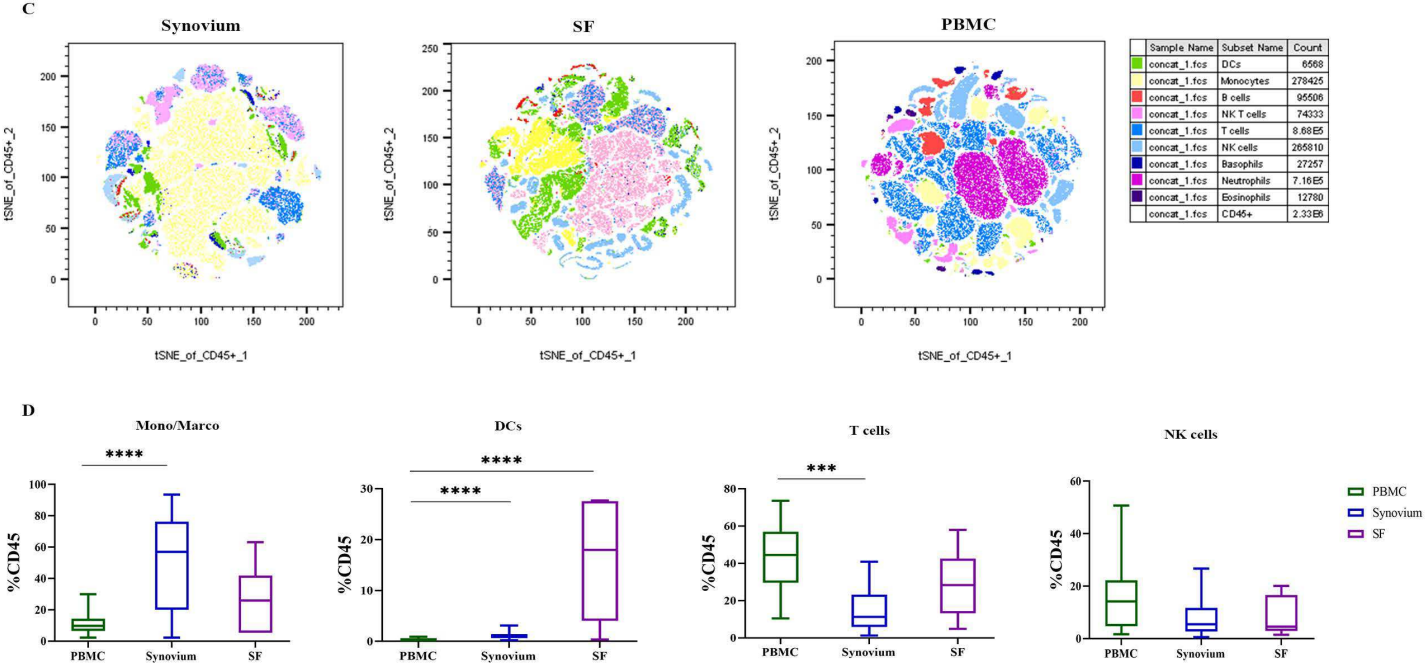
Figure 2.

A
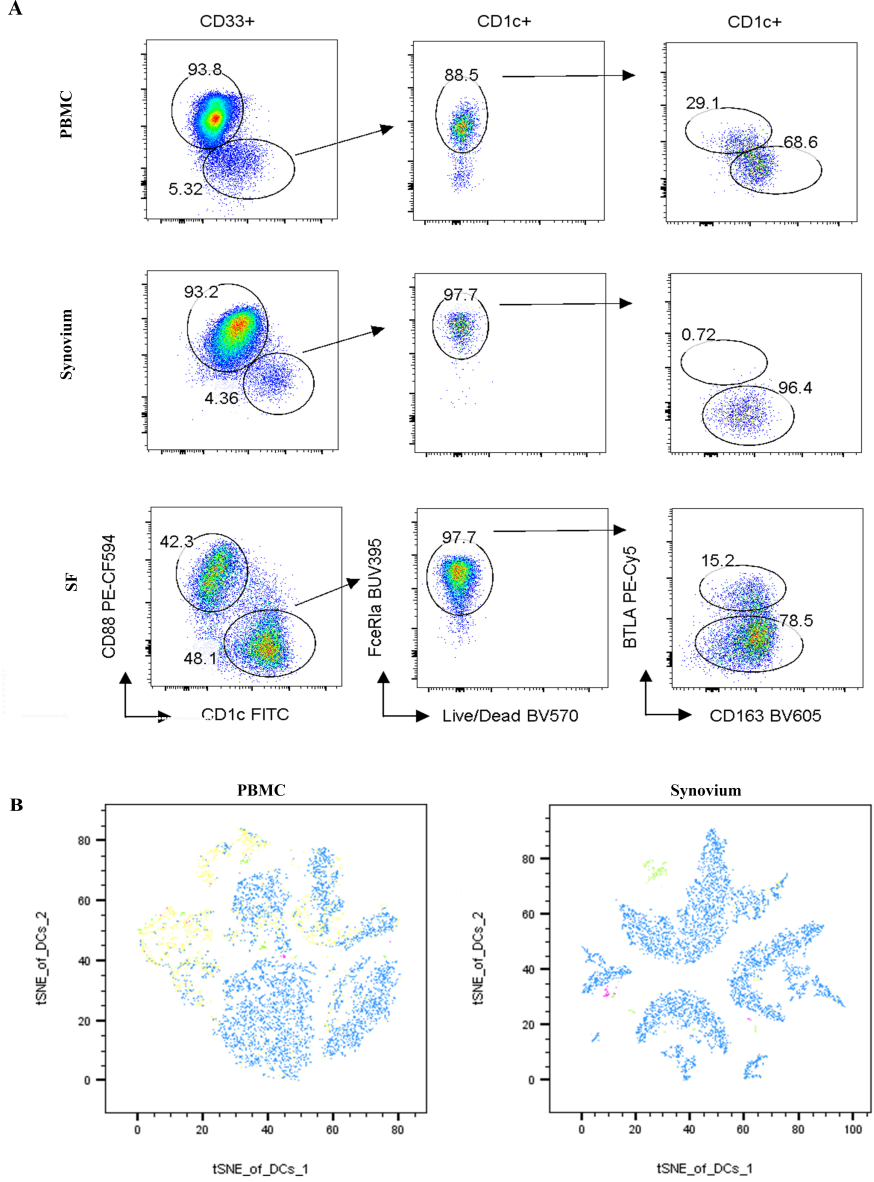
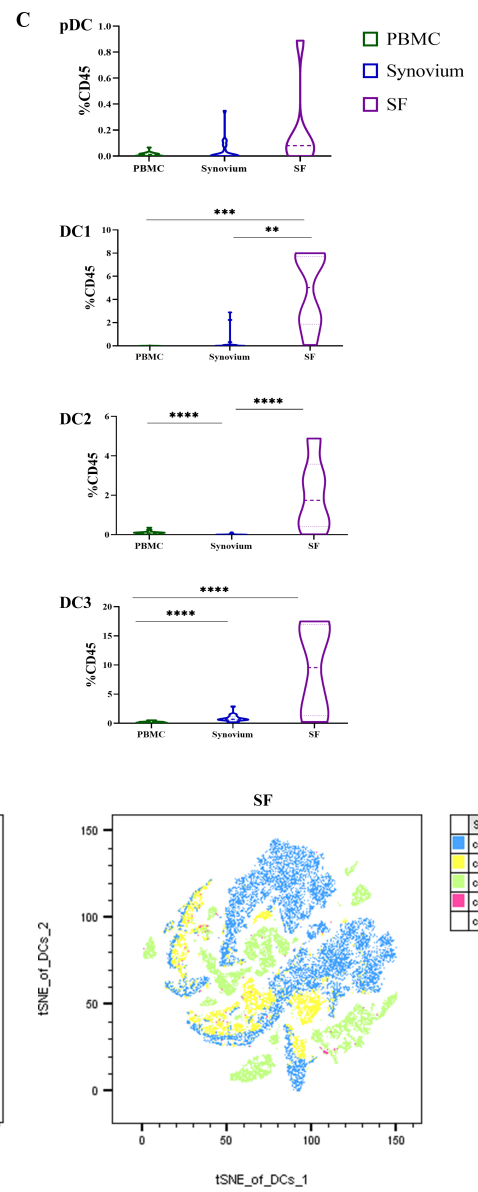

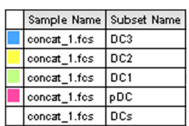


Figure 3.

A
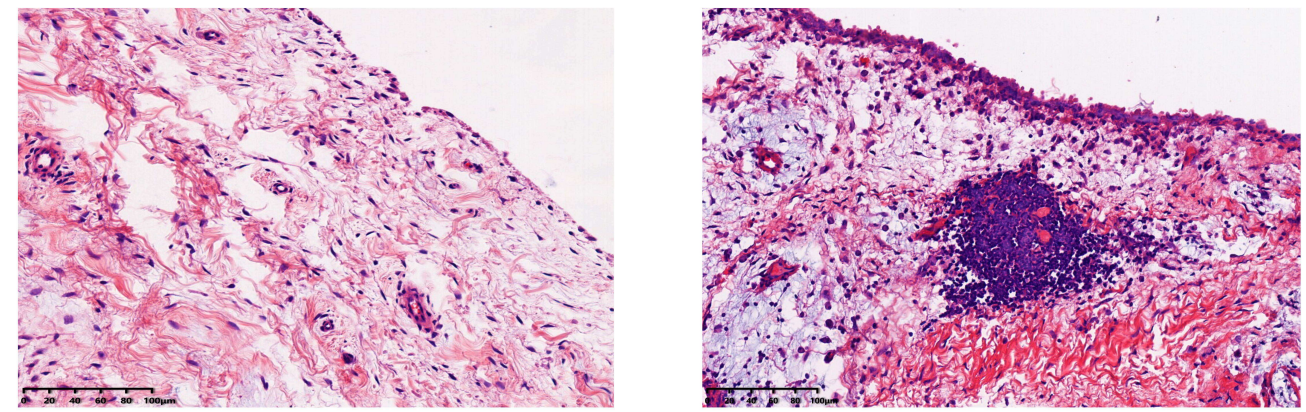

B
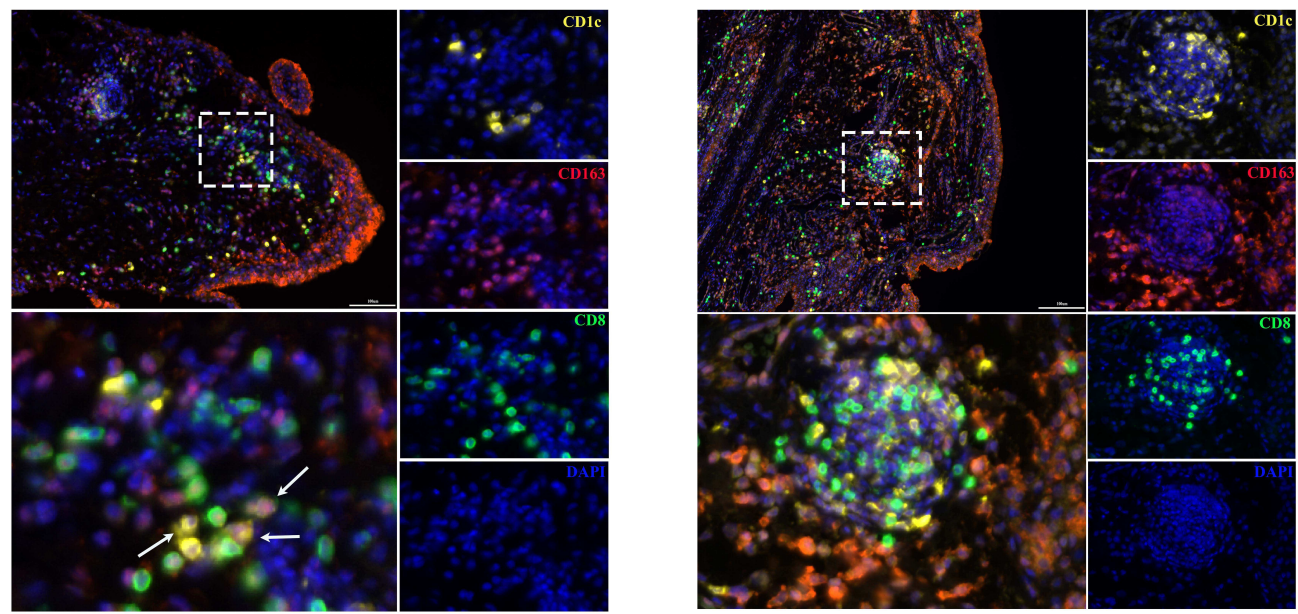

C
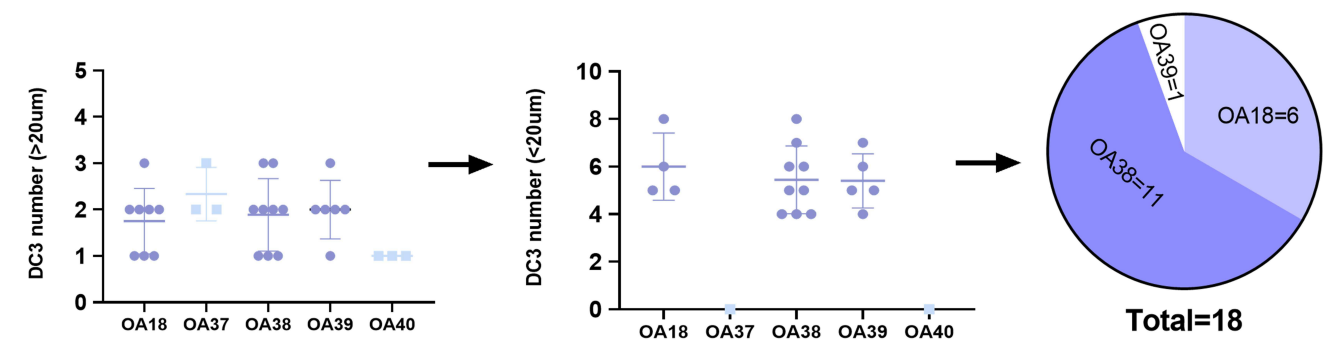

D

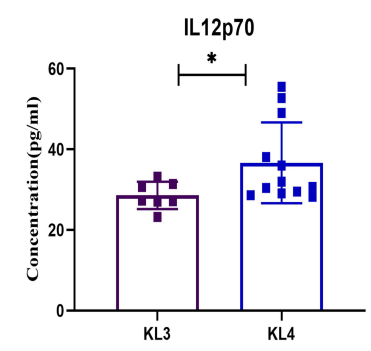

E

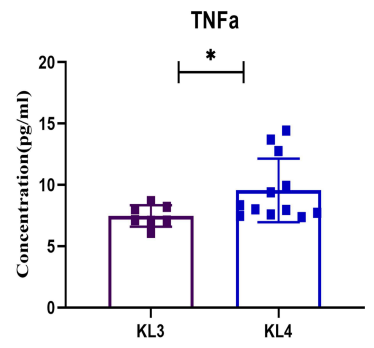

F

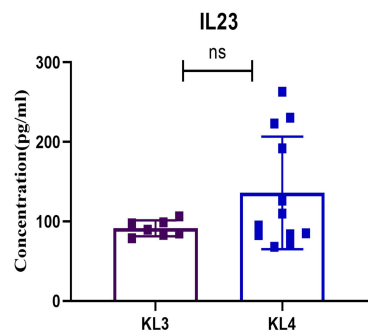




\section{Supplementary Files}

This is a list of supplementary files associated with this preprint. Click to download.

- SupplementalFigures.pdf 